\title{
La guerra fría desde la frontera México-Estados Unidos: entre codependencias y relaciones asimétricas, 1945-1975
}

\section{The Cold War from the US-Mexico Border: Between Codependencies and Asymmetric Relationships, 1945-1975}

\author{
Marco Antonio Samaniego López* \\ (D) http://orcid.org/0000-0003-4558-2342 \\ Instituto de Investigaciones Históricas \\ Universidad Autónoma de Baja California, México \\ samaniego@uabc.edu.mx
}

Resumen: El trabajo aborda el tema de cómo se constituye la frontera México-Estados Unidos durante la guerra fría. Se demuestra cómo inicia América Latina con la idea de plantear la pregunta de la simultaneidad o la diferenciación de los procesos ante la condición generada en la posguerra. Se demuestra que la construcción de esta no responde a los supuestos más difundidos por el gobierno de México, dado que las necesidades de coordinación llevaron a acuerdos y negociaciones que le dieron funcionalidad y base para la vida coti-

* Doctor en Historia por el Centro de Estudios Históricos de El Colegio de México. Líneas de investigación: La frontera México-Estados Unidos como construcción social. Hacia una nueva epistemología sobre las fronteras; Historia y memoria colectiva. Anarquismo contra liberalismo en la discursividad del Partido Liberal Mexicano.

cómo citar: Samaniego López, M. A. (2021). La guerra fría desde la frontera México-Estados Unidos: entre codependencias y relaciones asimétricas, 1945-1975. Secuencia (111), e1942. DoI: https://doi. org/10.18234/secuencia.v0i111.1942 
diana de los habitantes en ambos países. Paradójicamente, en esa misma zona, los procesos migratorios de mano de obra mexicana marcaron una situación de exclusión y represión. Por otra parte, Estados Unidos consideraba a su frontera sur como un posible acceso de agentes comunistas, por lo que crear obstáculos fue parte de sus políticas. Al mismo tiempo, la excepción fiscal dio pie a que el establecimiento de industria maquiladora se convirtiera en base de la economía fronteriza, producto de la expansión de las carreteras que tenían, como uno de sus objetivos, permitir el desalojo de las grandes ciudades.

Palabras clave: guerra fría; frontera México-Estados Unidos; cuencas internacionales; cooperación; conflicto.

Abstract: This paper addresses the issue of how the United States-Mexico border was constituted during the cold war. It shows how Latin America began, with the idea of raising the question of the simultaneity or differentiation of processes in the face of the condition created in the postwar period. It shows that its construction does not reflect the assumptions most widely publicized by the Mexican government, given that the need to coordinate led to agreements and negotiations that gave it functionality and a basis for the daily life of the inhabitants of both countries. Paradoxically, in that same area, the migratory processes of Mexican labor established a situation of exclusion and repression. Moreover, since the United States considered its southern border a possible means of access for communist agents, creating obstacles was part of its policies. At the same time, tax exemptions enabled the maquiladora industry to become the basis of the border economy, because of the expansion of highways, one of whose objectives was to permit the evacuation of major cities.

Keywords: cold war; Mexico-United States border; international basins; cooperation; conflict.

Recibido: 22 de enero de 2021 Aceptado: 2 de agosto de 2021 Publicado: 24 de septiembre de 2021

a guerra fría dejó huellas profundas en la segunda mitad del siglo xx en
el continente americano. El marco general es un mundo posterior al lan- 
zamiento de la bomba atómica en las ciudades de Hiroshima y Nagasaki, el 6 y 9 de agosto de 1945, respectivamente. A partir de la evidencia del potencial destructivo, las relaciones internacionales cobraron una nueva dimensión. El enfrentamiento entre Estados Unidos y la entonces Unión de Repúblicas Socialistas Soviéticas generó una tensión en una zona en que la relación entre dos países es estrecha y, al mismo tiempo, con asimetrías que son insoslayables para comprender la compleja región México-Estados Unidos. Economías distintas y con niveles muy diferentes en los mercados internacionales, pero codependientes en varios temas. Uno de ellos, que destacamos en este escrito, es el referente a las cuencas internacionales.

¿Es posible que en esta relación México tenga opciones de negociación ante estados Unidos, país que se fortaleció durante la segunda guerra mundial?, ¿se puede hablar de soberanía mexicana en espacios de frontera, cuando a nivel latinoamericano se aborda el periodo de la guerra fría como una relación que incidió de manera directa en el establecimiento de gobiernos militares, con profundas afectaciones a los derechos humanos?, ¿se puede hablar de cooperación entre México y Estados Unidos, mientras Chile, Argentina, Uruguay o Brasil enfrentaban gobiernos militares?, ¿es posible indicar que México incide en la extensa frontera, a pesar de las asimetrías innegables con su poderoso vecino?

Este escrito plantea que fue posible negociar y establecer una política coherente, pero no en todos los sentidos ni para todos los temas. Utilizamos esta diferenciación porque consideramos que permite ubicar la relación. Partimos de la idea que bajo el planteamiento de codependencia/negociación y asimetría/unilateral, como opuestos pero que se presentan de manera simultánea es una manera de contextualizar el periodo que permite resolver la paradoja de mutuos beneficios y de imposición unilateral. Existen numerosas teorías contemporáneas sobre la frontera (Agnew, 2005; Anderson, 1996; Bustamante, 1989; Strange, 2002); sin embargo, proponemos que la dicotomía planteada es una forma de ubicar una relación que en el espacio es cotidiana, intensa y contradictoria, dado que los recursos naturales y la propia condición fronteriza (seguridad nacional, saneamiento, aguas subterráneas) imponen necesidades específicas. Por ello, el eje de la historia global, como la guerra fría, se relaciona directamente con la propuesta de codependencia/ negociación y asimetría/unilateralidad.

Explicamos la propuesta de la siguiente forma. En los temas en que es conveniente para los dos países, la negociación tiende a ser mejor en relación 
directa a las condiciones de necesidad mutua. Además, la extensa frontera implica problemas diversos, por lo que las presiones de grupos organizados, empresas o las entidades federativas toman un matiz diferente. En este escrito lo demostramos en relación con los ríos internacionales, pero lo relacionamos con otros temas que obligan a pensar en varias fronteras. Un asunto que abordamos de manera relacionada es el referente a la mano de obra mexicana. $\mathrm{Si}$ bien no es nuestro tema central, lo destacamos para demostrar cómo en términos de manejo de las cuencas existe una relación que pasa por marcos institucionales formales, pero la mano de obra fue un tema en el cual los gobiernos mexicanos prestan poca atención, para demostrar la buena relación, sin ahondar en los problemas derivados de la violación de los derechos humanos y un manejo unilateral del problema por parte de Estados Unidos. ${ }^{1}$ Así, como veremos, los presidentes celebraron la construcción de grandes obras hidráulicas, pero no plantearon públicamente el tema de la mano de obra. Esta paradoja la extendemos a la propuesta de construcción de cercos en la frontera y el inicio de la expansión del sistema maquilador, que incrementó el poblamiento en zonas inmediatas a límite internacional del lado mexicano. Con ello pretendemos demostrar las varias fronteras, pero que coexisten dentro de la complejidad de la relación. Por ello, cerramos con un apartado que implica cómo los accidentes geográficos y las instituciones académicas son también parte de esta relación en la que, de acuerdo con la conflictividad, existe una relación codependiente/negociadora, pero que, en otros temas, sobre todo la migratoria, este se vuelve asimétrica/unilateral. Al referirnos a esta última, el país que aplica el criterio unilateral es Estados Unidos. Hasta el momento, no ubicamos un tema en el que suceda el caso contrario.

El límite fronterizo ha marcado la relación por el tema de la migración de mano de obra desde finales del siglo xix. Durante los años de 1942 a 1964, la estrategia de los dos gobiernos fue de abierta colaboración para trasladar de manera organizada a trabajadores mexicanos al vecino país del norte que se dedicaran a las actividades agrícolas. Sin embargo, al observar las propuestas y acciones de amplios sectores de Estados Unidos con respecto a la seguridad nacional, la presencia e incremento de la patrulla fronteriza, así como el lla-

${ }^{1}$ La creación del Centro de Estudios de la Frontera Norte de México (Cefnomex), posteriormente El Colegio de la Frontera Norte, de acuerdo con escritos de Jorge Bustamante Fernández, plantea una historia en la que el reconocimiento del problema ante las autoridades mexicanas fue una pugna de más de una década. 
mado a la acción militar en la extensa frontera, genera la dicotomía que hemos planteado. En efecto, los 3185 kilómetros de límite fronterizo representan un enorme reto para Estados Unidos, dada la extensión, la complejidad del espacio, así como las relaciones familiares, comerciales y turísticas que existen de manera recíproca. Es decir, ante el temor de que la zona pudiera ser utilizada por sus enemigos, así como por las ideas contrarias a la migración masiva, las acciones para proteger el espacio fronterizo se fortalecieron durante los años de la guerra fría. Así, por un lado, se instituyeron proyectos de orden material de gran envergadura -como las presas internacionales- mientras que, para ofrecer garantías de seguridad, se llevaron a efecto varios proyectos para intentar construir una separación que eliminara posibilidades de ingreso informal.

En este escrito demostramos que la lógica del espacio fronterizo entre México y Estados Unidos se constituyó una línea de desarrollo histórico que no corresponde a la relación México-Estados Unidos, que se observa desde los gobiernos centrales en numerosos trabajos académicos (Loaeza, 2016; Meyer, 2010). Es decir, los imperativos geográficos y las necesidades de las ciudades y áreas agrícolas vinculadas por las obras hidráulicas en las cuencas internacionales, generó un conjunto de acuerdos, mismos que, para darles continuidad, llevó a la necesidad de cooperación permanente (Bustamante, 1999; Samaniego, 2012). Esta idea se opone a otras condiciones que se establecen por razones de seguridad nacional para Estados Unidos, sobre todo a las acciones de militarización demandadas por diferentes analistas que consideraron el temor de su frontera sur como una posible penetración para los ataques de sus enemigos. ${ }^{2}$ Así, una de las ideas más citadas, realizada por el internacionalista Mario Ojeda, es que México tuvo ámbitos de acción permitidos por Estados Unidos, pero dentro de ciertos límites, que generaron la idea nacionalista de una política exterior autónoma que defiende la postura de la no intervención y la libre autodeterminación de los pueblos (Meyer, 2010; Ojeda, 1978). Si bien consideramos que la tesis es sostenible, deseamos agregar que Estados Unidos se ve obligado a negociar dado que las demandas de su población no son homogéneas. Mientras algunos sectores exigían mayor dureza con respecto a la migración de mano de obra, otros, sobre todo

${ }^{2}$ Esta condición se puede abordar desde finales del siglo xix y principios del xx. Sobre todo, para el caso de la migración japonesa, misma que se observa como una amenaza para la costa oeste. 
quienes dependían de una buena relación en las zonas ligadas a la frontera, buscan llegar a acuerdos dada la mutua conveniencia.

Partimos de una base de relación en que se construyó codependencia en la que -a pesar de las asimetrías y la necesidad de vincularse y administrar en lo posible el extenso límite-provocó la creación de instituciones, así como acuerdos locales de orden formal e informal ${ }^{3}$ que generaron una manera de vincularse -a pesar de que la relación fuera tensa entre los gobiernos centrales y de que existían mecanismos previos de los que había mutuos beneficios-. Sin duda, la institución más importante es la Comisión Internacional de Límites y Aguas (CILA), misma que busca articular la construcción del espacio en ambos lados de la frontera. La sección mexicana depende de la Secretaría de Relaciones Exteriores; la estadunidense, del Departamento de Estado. ${ }^{4}$ Su carácter binacional y la importancia de las decisiones que se toman de manera conjunta incidió de manera directa en la constitución de zonas agrícolas, poblaciones y ciudades, así como en la administración del recurso agua, mismo que en condiciones de aridez resulta fundamental para explicar el poblamiento y la proyección de futuro con base en los tratados que han consolidado al organismo (Samaniego, 2006, 2012). Cabe señalar que la mencionada institución, hasta la fecha, enfrenta numerosos problemas, varios de ellos derivados por el crecimiento de las ciudades en ambos lados de la frontera, situación que ha provocado nuevas necesidades de articulación, sobre todo por acciones encaminadas al saneamiento. De igual forma, sobre las aguas subterráneas compartidas existen vacíos en la interpretación para su aprovechamiento (Hatch, 2017).

La existencia de esta institución, desde nuestro punto de vista, ubica en el centro de la discusión una de las tesis más utilizadas por la diplomacia mexicana en los años de la guerra fría: la no intervención y la libre autodeterminación de los pueblos. ¿Cómo generar una política exterior en torno a la soberanía cuando las decisiones sobre el espacio fronterizo fueron resultado

${ }^{3}$ Hacemos la diferenciación de acuerdos formales e informales, dado que se generan acuerdos de diversa índole que, aunque se establecen ciertas prácticas, no llegan a ser acuerdos reconocidos por las autoridades de cada país. Por otra parte, sí existen modalidades que llegan a formalizarse.

${ }^{4}$ La institución surgió en 1889, debido a las diferencias que se presentaron con respecto al límite internacional. Las facultades de esta se han modificado en relación directa con las nuevas necesidades, aunque ha quedado claro que sus temas fundamentales son los límites, el agua superficial y subterránea, así como temas medioambientales. 
de consensos/disensos en la CILA?, ¿cómo sostener que la política exterior mexicana es una -y sólo una-cuando los procesos en torno al espacio fronterizo están en relación directa a obras que se construyen el país vecino o como efecto de políticas empresariales que se benefician de las condiciones generadas por el límite territorial?, ¿qué implica la existencia de una institución de orden binacional, que para su accionar requiere de cumplir con la aprobación de su contraparte?

Sobre esto último, destacamos que las ciudades fronterizas (Matamoros, Ciudad Juárez, Mexicali, Nogales, Tijuana, por mencionar algunas) están ligadas directamente a los conflictos laborales generados en la posguerra en Estados Unidos. La industria maquiladora, que tiene como fundamento la excepción fiscal a la introducción de insumos para luego retornar al país de origen, como bienes manufacturados, es un motor de crecimiento poblacional en los años aquí abordados. Las ciudades crecieron con base en la forma en que empresarios de diversas nacionalidades -destacan estadunidenses, japoneses y coreanos- trasladaron parte de sus procesos productivos, entre otras razones, como medida para debilitar las organizaciones de trabajadores en Estados Unidos y aprovechar la mano de obra mexicana a menor costo (Carrillo, 1985). Dicha industria se desarrolló con base en los acuerdos realizados por la CILA, mismos que permiten que se obtenga agua para zonas que bajo condiciones regionales sería imposible obtener. La migración provocada por este crecimiento es un factor relevante para explicar las cadenas de migrantes hacia Estados Unidos, así como el incremento de problemas de saneamiento provocadas por la urbanización acelerada y sin control en la mayoría de los casos.

Así, mientras México se presentó ante el mundo y Latinoamérica como un país que tenía como centro ideológico el nacionalismo revolucionario, negoció con Estados Unidos la realización de obras conjuntas en el límite fronterizo y acordó entregar/recibir recurso hídrico que es vital para la existencia y crecimiento de zonas agrícolas, así como sustento de la industria maquiladora. Incluso, acordó enviar mano de obra a pesar de que, durante más de una década, existió otro programa, conocido como la Operation Wetback, que consistió en la deportación sistemática de mexicanos, bajo el argumento de que estos no cumplieron con los requisitos establecidos por el Programa Bracero. Además, México permitió un régimen de excepción fiscal que beneficiaba claramente a empresarios extranjeros, lo que era una contradicción a los programas fomentados por la Comisión Económica para 
América Latina y el Caribe (CEPAL), con la implementación de regímenes de sustitución de importaciones. Incluso, uno de los máximos orgullos del régimen autodenominado revolucionario era el desarrollo de la industria nacional bajo el esquema planteado por la CEPAL y que estaba en concordancia con otros países de América Latina (Cárdenas, 1996). En la década de 1970, sectores académicos ubicaron la explicación sobre la industria maquiladora en la teoría de la dependencia, con un bajo margen de acción por parte de México (Ojeda, 1976, 1978).

Así pues, el concepto de América Latina se aplica generalmente desde México hacia el sur ${ }^{5}$ pero, ¿cómo inicia el uso del espacio y en este caso qué implicaciones tiene el compartir un recurso natural de fundamental relevancia para el poblamiento y desarrollo de la zona?, ¿es un límite internacional que separa, en realidad, dos mundos diferentes?, ise puede aplicar el concepto de codependencia, cuando, el uso de la violencia por parte de Estados Unidos fue parte fundamental de los regímenes autoritarios en el Cono Sur y el Caribe?, ¿cómo debemos identificar, por tanto, la política internacional mexicana, desde una condición de codependencia/negociación y asimetría/unilateral en su límite con el país que con sus intervenciones incidió en el derrotero totalitario que se impuso en países del Cono Sur o Centroamérica?, ¿es el inicio de Latinoamérica, una excepción conveniente? El historiador inglés, Leslie Micheal Bethell, al abordar si Brasil era parte o no de América Latina, indica que aun en el siglo xx numerosos intelectuales lo ponían en duda (Bethell, 2016, pp. 118-126). Aquí sostenemos que la historia del límite fronterizo es un factor que poco incide en la construcción del imaginario nacional mexicano, pero que es de gran peso para entender una relación con el vecino que incide en todo el continente y que, paradójicamente, requiere de un buen entendimiento en el límite territorial. Apuntamos que en este escrito abordamos el periodo de 1945 a 1975, dado que la complejidad de la relación nos obliga a tratar de explicar el proceso tomando en consideración varios temas, mismos que ubicamos como indispensables para comprender cómo se ha construido un espacio donde la relación es paradójica, contradictoria y, a su vez, funcional para diversos actores en cada una de las dos naciones. Apuntamos que la fecha de 1975 es tomada como referente debido a que se concluye con uno

${ }^{5}$ Existen autores que ponen en duda el uso del concepto dadas las diferencias que existen en lo que se denomina comúnmente como América Latina. Algunos de ellos, Mignolo (2005) y Moya (2016). 
de los problemas que surgieron, como es la salinidad de los pozos de Wellton-Mohawk, Arizona, pero esto no implica que no hubiera otros procesos simultáneos que pueden ubicarse en la relación que abordamos en el presente escrito. Lo que aquí planteamos tiene como marco la guerra fría, desde el lugar en que, desde la construcción del concepto de América Latina, ${ }^{6}$ presenta una idea de diferenciación pero que, como veremos, tiene sus límites y se constituye, independientemente del discurso, en una relación conveniente.

\section{LA POSGUERRA: REFERENCIAS NECESARIAS}

El fin de la segunda guerra mundial estuvo lejos de traer la calma después de la tormenta. El mundo se encontró en una nueva condición de alarma por el uso y la experimentación de armas atómicas. La Unión de Repúblicas Socialistas Soviéticas (URSS), en 1949, logró seguir los pasos de su enemigo ideológico y pronto incrementó su capacidad militar. Se inició una carrera armamentista en la que ambos bloques comenzaron a desarrollar las primeras bombas termonucleares basadas en la fusión de átomos. Estados Unidos realizó su primera prueba en noviembre de 1952, a la que la URSS respondió en agosto de 1953 con el lanzamiento de su propia bomba en la base de Kazajistán. Si bien hubo acercamientos entre la URSS y Estados Unidos, estos pronto incrementaron sus diferencias, sobre todo cuando las intervenciones en otros países implicaron la búsqueda de contrapesos que legitimaran sus respectivas posiciones ante los órganos internacionales, como la Organización de Naciones Unidas (Powaski, 1998).

Harry S. Truman fue el promotor de la política de contención, término ideado en 1947 por George F. Kennan, quien planteó la necesidad de eliminar la posibilidad de que el comunismo se expandiera en el mundo. Truman, ante el Congreso de su país, el 12 de marzo de 1947 afirmó que la política de Estados Unidos era apoyar a los pueblos libres que estaban resistiendo intentos de agresión de minorías armadas o presión exterior. En septiembre de ese año se creó la Agencia Central de Inteligencia (CıA, por sus siglas en inglés), organi-

${ }^{6}$ Al indicar desde el concepto de América Latina, lo señalamos por la relación que existe entre la invasión a Nicaragua realizada por William Walker y el concepto que surgió a partir de ese proceso. La relación se debe a que el mismo personaje, previamente, había intentado formar una república independiente en 1853-1854 en Sonora y Baja California, en el entorno de las negociaciones por adquirir nuevos territorios, encabezada por James Gadsden. 
zación que resultó la punta de lanza en la coordinación de acciones intervencionistas en las décadas siguientes tanto en Europa como en América Latina (Saunders, 2001). En 1953 realizaron la primera operación encubierta -Ajax Project- para derrocar al primer ministro iraní, Mohammad Mosaddegh y apoyar al monarca Mohammad Reza Pahlavi, quien respondía a los intereses de Inglaterra y Estados Unidos.

El triunfo del comunismo en China en 1949 y el avance de Corea del Norte, con apoyo chino y soviético en 1950, sobre Corea del Sur, prendieron las alarmas del gobierno estadunidense que veía con temor la opción comunista. Para varios grupos al interior de Estados Unidos Truman resultaba débil, a pesar de la orden de lanzar las bombas atómicas (Gaddis, 1972; LaFeber, 1972; Leffler, 1992).

En ese contexto, la CiA organizó en 1954 la intervención de Estados Unidos para destituir al presidente guatemalteco Jacobo Árbenz. El cuidado de la relación con Estados Unidos marcó la actuación del presidente mexicano Adolfo Ruiz Cortines, lo que generó posiciones encontradas entre la elite política nacional (Loaeza, 2016, pp. 727-728). Algunos sectores disidentes realizaron intensas campañas en contra de la intervención. En la X Conferencia Interamericana de Caracas, el secretario de Estado, John Foster Dulles, disertó sobre la necesidad de una alianza hemisférica, argumentando que cualquier injerencia del comunismo internacional era inadmisible en el continente. El secretario de Relaciones Exteriores de México, Luis Padilla, argumentó en defensa de la libre autodeterminación de los pueblos y la no intervención, aunque no en defensa explicita de Árbenz. En la resolución, México y Argentina se abstuvieron de votar, Guatemala se opuso y los otros 17 países del continente la aprobaron.

La presencia de la URSS en México tuvo varios episodios. A finales de la década de 1950 iniciaron varios contactos, en el marco de la coexistencia pacífica, concepto difundido por Nikita Khrushchev. En 1961 formó el Instituto de América Latina de la Academia de Ciencias, mismo que daba sustento a la pretensión de dar apoyo a las economías latinoamericanas (Pettinà, 2016). Se creía que estas podrían seguir los pasos de la Unión Soviética si conocían los beneficios sociales producto de la intervención del Estado en la economía. Por ello, en parte, las propuestas de la CEPAL fueron vistas por amplios sectores como un avance de las ideas comunistas. Sin embargo, como apuntamos anteriormente, en el extenso límite entre México y Estados Unidos, el proyecto que iniciaba formalmente en la década de 1960 se basaba en 
la excepción fiscal: la industria maquiladora ligada estrechamente a la producción estadunidense. Además, una larga tradición de libertad comercial en el que las aduanas actuaban sólo en determinados espacios, dejando enormes zonas con posibilidades de comercio sin control.

La revolución cubana fue un acontecimiento que cimbró el escenario de la guerra fría. Mientras John F. Kennedy planteó la Alianza para el Progreso, con la intención de establecer una política de colaboración continental, la relación de Fidel Castro con la URSS planteó dudas que incrementaron el temor de un ataque (Pettinà, 2018, pp. 98-99). La crisis de los misiles de octubre de 1962 fue el momento de mayor tensión (Mikoyan, 2012). El presidente Adolfo López Mateos estuvo a favor de Estados Unidos en el bloqueo a la isla, así como para exigir el retiro de los misiles soviéticos. Poco después, en 1964, con Lyndon B. Johnson como presidente, López Mateos, en la novena reunión de consulta de la Organización de Estados Americanos (OEA) votó en contra de la resolución de romper relaciones con la isla (Meyer, 2010). Con ello, México tuvo un argumento en la línea sobre el principio de no intervención y libre autodeterminación de los pueblos. Sin duda, uno de los episodios más referidos sobre la política internacional de nuestro país, siempre y cuando no se observe el proceso de construcción del espacio fronterizo al que aludimos en este escrito.

Los años siguientes se pueden resumir para el caso estadunidense entre la tensión de indicar que el triunfo de la guerra de Vietnam estaba a la vuelta de la esquina y la condición de que dicha conflagración estaba siempre en un punto más difícil de lograr el objetivo (Kissinger, 2003; Powaski, 1998). Las atrocidades cometidas por las tropas estadunidenses provocaron que la intervención se convirtiera en una paradoja en la que, las críticas a la guerra, era alimentadas en buena medida por los propios soldados que denunciaron los crímenes (Prados, 2009; Summers, 1981). El presidente Lyndon B. Jonhson, a pesar del éxito de sus programas sociales, se vio imposibilitado de plantear su reelección dado que el tema Vietnam era un fracaso que marcó su administración.

Sin embargo, las intervenciones en América Latina tuvieron particular importancia en los años de dominio de Richard Nixon y Henry Kissinger. En 1967, el secretario de Defensa estadunidense afirmó que el objetivo principal para Latinoamérica era el desarrollo de fuerzas militares y paramilitares locales para asegurar la seguridad interna. Estados Unidos invirtió en el fortalecimiento de los ejércitos del Cono Sur. La Escuela del Ejército de las Américas (United States Army School of the Americas) en Panamá, fue el princi- 
pal centro de adiestramiento. En sus cursos incluyeron el peligro del avance rojo. Se enseñó a realizar torturas o fabricar bombas con elementos caseros. En Paraguay, entre los Archivos del Horror, localizados en 1992, se encontró un manual que instruía sobre cómo mantener vivas a personas torturadas con electricidad. En Fort Gulick, Panamá, funcionó el centro de operaciones contrainsurgentes a través de la red de telecomunicaciones del gobierno de Estados Unidos (Paredes, 2004; Selser, 1982). En 1975, la Operación Cóndor fue el nombre con el que Kissinger le dio continuidad a una política de intervención que favoreció dictaduras militares que dejaron miles de muertos y desaparecidos.

\section{LÍMITE FRONTERIZO Y TECNOLOGÍA}

A 150 kilómetros del límite internacional entre México y Estados Unidos, en el lugar conocido como Jornada del Muerto, cerca de Alamogordo, Nuevo México, se realizó en julio de 1945 la primera prueba nuclear. Las narrativas sobre el material nuclear que se desparramaba en el aire, así como los daños ambientales, generaron en los habitantes de Nuevo México y Texas, en Estados Unidos, y Chihuahua, en México, un sentido contrario a la "seguridad" nacional. El Proyecto Manhattan, a pesar de la secrecía con que se desarrolló, tuvo un espacio de prueba que mostró una cara publica que generó un sentido de inseguridad en ambos lados del límite internacional (Hodesson, Henriksen, Meade y Westfall, 1993). La prueba Trinity implicó la movilización de miles de hombres a una zona desértica, considerada una opción para el ejercicio militar. Los directivos del Proyecto Manhattan, por desconocer los posibles efectos de la detonación, solicitaron al gobernador de Nuevo México que preparara varios escenarios de catástrofe. Podía desaparecer toda la entidad, alguna población, e incluso algunos de los científicos consideraron que podría presentarse el holocausto. La detonación despertó a los habitantes de El Paso, Texas, inmediato a la frontera con México, en Ciudad Juárez, Chihuahua.

De manera paralela, los Senados de los dos países discutían un proyecto de tratado que, firmado originalmente en febrero de 1944, también había sido negociado en secrecía, sobre todo para no complicar su aprobación por la oposición encabezada por el estado de California (Enríquez, 1976; Hundley, 1963; Samaniego, 2006). Dicha entidad, clave en las operaciones de guerra debido a su posición en la costa del Pacífico, reclamaba que a México se le entregaba demasiada agua en el Tratado de Aguas Internacionales, cuya aprobación 
estaba en discusión en el Senado estadunidense. Dicho documento sancionaba una relación a perpetuidad entre ambos países, mismos que, apegados a principios de colaboración, acordaron la distribución de las aguas de los ríos Bravo y Colorado. De acuerdo con el Tratado de Guadalupe-Hidalgo de $1848,{ }^{7}$ ambos cuerpos de agua eran considerados internacionales por ser factores clave en la delimitación territorial. Como puede observarse en el mapa 1, el río Bravo delimita claramente ambos países. Sin embargo, de Ciudad Juárez hacia el oeste, la frontera es de índole terrestre, pero, sobre todo, en condiciones de aridez que generan hasta nuestros días tensiones por la falta de agua.

Dicho tratado involucró a estados muy lejanos al límite territorial como son Wyoming, Utah, Colorado o Nevada, así como a los fronterizos de Arizona, California, Nuevo México y Texas. Para el caso mexicano involucró en diferentes dimensiones a los estados de Sonora, Chihuahua, Nuevo León, Coahuila y Tamaulipas, así como al entonces Territorio Norte de la Baja California. El acuerdo fue el resultado de la inversión en obras hidráulicas de gran dimensión, sobre todo de Estados Unidos, y de la aplicación conveniente para los dos países del principio de primera apropiación en su relación con el uso benéfico, mismo que fue la base para acordar la cantidad de agua que le correspondía a cada nación (Samaniego, 2006, 2012, 2018). Por considerar que las necesidades de su entidad eran mayores, los californianos argumentaban que el tratado los limitaba en su desarrollo futuro, sobre todo por el contexto de guerra en el que estaban inmersos. Como contraparte, Texas, que recibe agua de afluentes mexicanos del bajo Bravo, apoyaba la firma del tratado y realizaba campañas para su aprobación, mientras California lo hacía en sentido opuesto.

Aquí apuntamos que dicho tratado fue un eje que permitió estructurar una relación de codependencia, misma que se había iniciado con el siglo xx, pero que, con la sanción correspondiente, marcó un derrotero a seguir en los años siguientes. Dado que es parte fundamental para el periodo aquí tratado, lo ubicamos en lo que hemos denominado codependencia/negociación, con efectos de suma relevancia para la construcción del espacio fronterizo, dado que incide de manera directa en la vida de millones de personas en ambos países, profundamente vinculado al tema de la existencia/destrucción de ecosistemas.

${ }^{7}$ El nombre oficial es Tratado de Amistad, Límites y Arreglo Definitivo entre los Estados Unidos Mexicanos y los Estados Unidos de América. 
Mapa 1. Ríos internacionales entre México y Estados Unidos

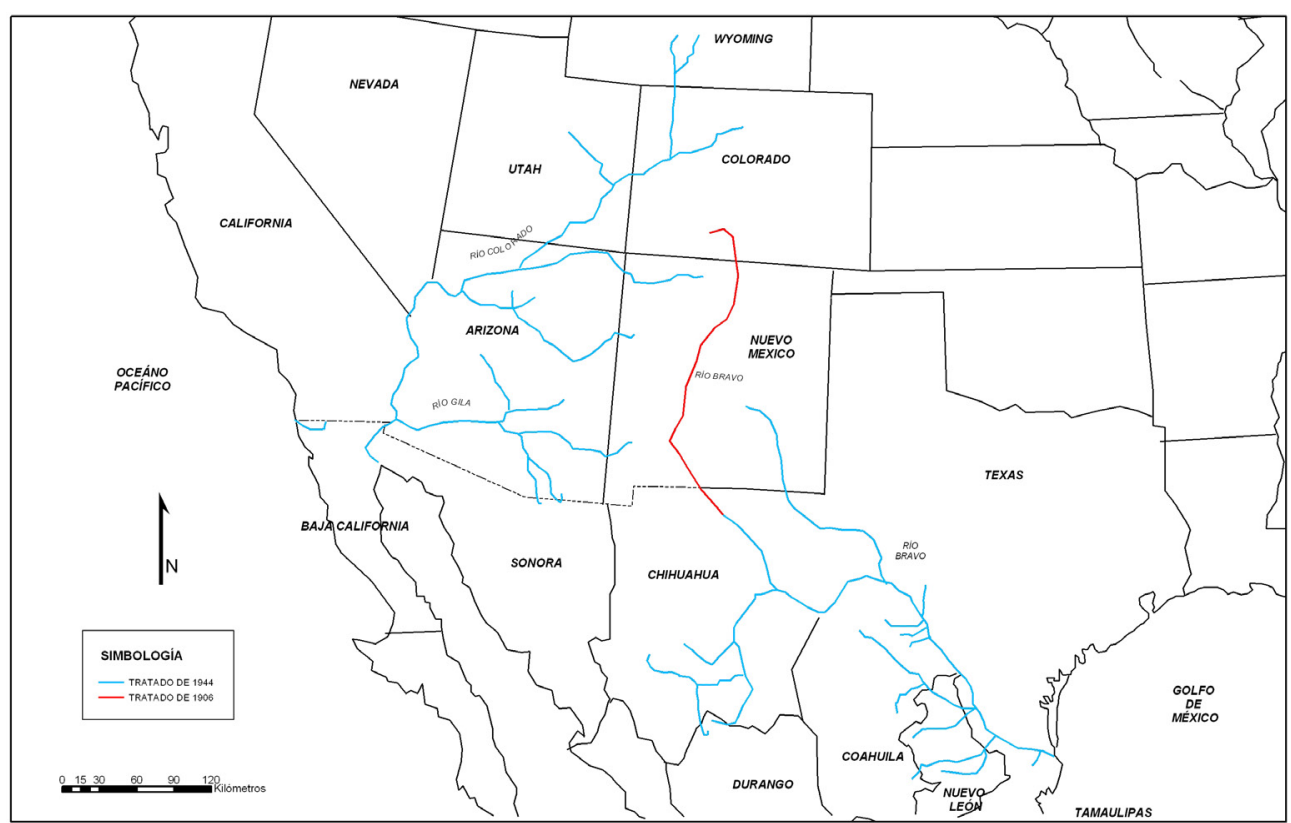

Fuente: Samaniego, 2006, p. 17. 
El documento estableció una ruta crítica que permitía garantizar inversiones, así como abasto de agua a zonas agrícolas y áreas urbanas en ambos lados de la frontera. Marcó la pauta en la distribución del recurso para los estados que están alejados del límite territorial, como Wyoming, Utah o Colorado -entidades en las que se origina el río Colorado como producto de las nevadas-e incidió en el respeto de acuerdos previos entre las entidades estadunidenses que tuvieron una intensa lucha en los años precedentes. Para ejemplificar la relación aquí expuesta, señalamos que los actores políticos de los tres órdenes de gobierno se articularon -con dificultades y diferenciaspara que, en Tijuana, en el estado de Baja California, se planeara su desarrollo económico a largo plazo, como el sistema maquilador, con el recurso hídrico que proviene de entidades tan lejanas como los mencionados estados de Wyoming, Utah y Colorado.

Así, el límite involucra de manera formal 3185 kilómetros, pero las cuencas internacionales obligan a pensar que la codependencia involucra áreas que son elementos clave para la vida humana y los ecosistemas. Así, por ejemplo, el río Conchos, principal afluente del Bravo en el lado mexicano, tiene una longitud de poco más de 900 kilómetros, con una superficie de cuenca de $62881 \mathrm{~km}^{2}$. El Colorado, con numerosos afluentes, tiene alrededor de 2334 kilómetros de longitud y una superficie de cuenca de $629100 \mathrm{~km}^{2}$. Aquí sólo mencionamos estas por ser centrales en los términos del documento, pero que ayudan a ilustrar la construcción de un sistema, a perpetuidad en términos del tratado, del cual dependen numerosas ciudades, zonas agrícolas y presas internacionales que producen energía eléctrica para ambos países.

Como eje de análisis, en una cuenca sucede una gran cantidad de eventos de manera simultánea. Ribereños que extraen agua para usos mineros o agrícolas, ciudades que gracias a obras hidráulicas obtienen recurso a grandes distancias del cuerpo de agua, como sería el caso de Los Ángeles, California, o Denver, Colorado, en Estados Unidos, o Tijuana y Tecate, en Baja California, México. De igual forma, zonas que reciben energía eléctrica de las presas Amistad y Falcon, como Laredo, Texas, o Nuevo Laredo, en Tamaulipas. También, aquí se incluyen zonas agrícolas que aprovechan el agua que recorre grandes distancias, como el valle de Matamoros en Tamaulipas, donde el recurso que ha sido utilizado en otras partes de la cuenca vuelve a ser aprovechado para la producción agrícola. En sentido contrario, se despojó a comunidades, particularmente indígenas de ambos lados de la frontera, de la posibilidad de mantener sus prácticas culturales, además de modificar de manera 
definitiva ecosistemas desérticos y semidesérticos. Bajo la idea de ganarle tierras al desierto, parte de este fue modificado para sostener a las áreas agrícolas y ciudades que se desarrollaron en los años siguientes (Pisani, 1992, 1996).

Los efectos del tratado de 1944 son numerosos por esta amplitud de actos de relación cotidiana que explican la construcción de un espacio fronterizo que se vuelve operativo y, como ya apuntamos, en la relación codependencia/negociación. ${ }^{8}$ Para los efectos de este escrito, destacamos que la firma en el contexto del inicio de la guerra fue un marco de referencia para los años siguientes. Obligaba a los dos países a construir dos presas internacionales para los cual se requería total cooperación de ambos lados, no sólo para su erección en el Bravo, sino para el manejo permanente. El tratado dio la posibilidad de una presa que era conveniente sólo para México, como fue la Anzaldúas, y que se aprobara por ambos países; con ello, era posible regular la corriente y abrir más tierras al cultivo. Para los texanos significaba garantizar agua de los afluentes mexicanos y conducirla, luego de producir energía, al denominado Valle Mágico, en el sur del estado; para las entidades superiores de la cuenca del Colorado -Nuevo México, Utah, Colorado, Wyoming-, garantizar el abasto a futuro, dado que sus proyectos apenas estaban iniciando. Para las entidades inferiores de la cuenca como California, representaba alimentar las ciudades que crecían en la costa, sobre todos Los Ángeles y San Diego. ${ }^{9}$ Arizona, por su parte, se mantuvo en una relación conflictiva con California, dado que consideraba injusto que los californianos fueran los más beneficiados con un cuerpo de agua al que no aportaban afluentes importantes. La pugna entre estas entidades es particularmente importante tanto en años precedentes al tratado como en los años de la guerra fría. Sin embargo, por las particularidades del tema no lo abordamos en este escrito.

De manera simultánea, en lo correspondiente a asimetría/unilateral, fueron constantes las propuestas de erigir obstáculos físicos para el cruce de mano de obra y posibles enemigos hacia Estados Unidos en la extensa fron-

${ }^{8}$ Bajo esta línea, este texto ha sido escrito desde la ciudad de Tijuana, Baja California, México, con el uso de agua para las actividades cotidianas, que responde a dicha construcción del espacio fronterizo, al que estamos ligados alrededor de 60000000 de personas en toda la cuenca de los ríos Bravo y Colorado.

${ }^{9}$ Las disputas entre California y Arizona iniciaron desde el pacto de Santa Fe, de 1922. Las intenciones de ambas entidades se trasladaron a demandas judiciales que se realizaron durante cuatro décadas, cuando en 1963 se tomaron decisiones que favorecieron parcialmente a Arizona. Aun así, las diferencias continuaron por las pretensiones de California de quedarse con más agua, situación que hasta la fecha prevalece. 
tera. La tensión de la guerra fría, con sus múltiples especulaciones sobre ataques de diverso tipo, así como la presencia evidente de organizaciones comunistas y una visión excluyente sobre quien debía ser parte de la nación, llevaron a incrementar proyectos y disposiciones formales para la erección de una barda que limitara los cruces informales. Debemos tomar en consideración que los debates sobre la discriminación y su práctica cotidiana eran muy intensos en varias entidades, particularmente en Texas (Menchaca, 2011; Orozco, 2009; Zamora, 2003)..$^{10}$

El 4 de agosto de 1942 se firmó el convenio de trabajadores huéspedes mexicanos en Estados Unidos, conocido generalmente como el Programa Bracero. Bajo los términos del acuerdo, la Farm Security Administration era la encargada de contratar a los mexicanos, a quienes debería de transportar a los centros de trabajo en condiciones de vida digna. Sin embargo, por la dificultad de los trámites, así como por la necesidad de la mano de obra en las zonas agrícolas, cumplir con los acuerdos formales provocó que se mantuviera la migración indocumentada. En un estimado general, alrededor de 4500000 trabajadores mexicanos estuvieron amparados en este acuerdo, mismo que tuvo varias modificaciones (Chacón, 2011; Munguía, 2015). A la par de ello, en 1942 se firmó el tratado comercial con Estados Unidos, mismo que estuvo vigente hasta 1947 y reactivó el flujo de varios productos, entre ellos el petróleo (Avella, 2016, 2018).

A pesar de ello, la frontera asimétrica/unilateral se reforzaba con la construcción de barreras, a partir de la Western Land Boundary Fence Project. Los antecedentes de este proyecto pueden rastrearse a la década de 1930; aquí sólo apuntamos que desde la guerra mundial los agentes de la patrulla fronteriza reclamaban mayor inversión y equipo, tema que se reflejó a finales de la década de 1940 con el avance del proyecto de construir la barda (Lytle, 2010). La sección estadunidense de la CILA, actuando de manera unilateral sin tomar en cuenta a la parte mexicana, se vinculó con otras agencias estadunidenses para responder a esta demanda y se inició la construcción de una malla que dividió varias poblaciones fronterizas que tradicionalmente estaban separa-

10 El caso Plessy vs Fergurson de 1896 legitimó la segregación racial entre afroamericanos y "blancos", por lo que el acceso a escuelas, albercas, cines, por mencionar algunas, estaba limitado. En los autobuses, los afroamericanos debían trasladarse en la parte trasera. La regla de "separados pero iguales" no incluía expresamente a los mexicanos, pero se aplicaba por extensión. En los años de la guerra fría, esta pugna generó numerosos movimientos sociales y sin duda hubo logros importantes en términos de derechos civiles. 
das por una calle, como Nogales, Sonora, y Nogales, Arizona, o Mexicali, Baja California, y Calexico, California (Álvarez, 2019). Sin embargo, el proyecto tuvo varias etapas dado que hubo resistencias locales, desacuerdos entre los participantes y falta de recursos. De manera paralela, la patrulla fronteriza instaló torres que desde el límite territorial les permitían ver hacia territorio mexicano y observar los movimientos de quienes pretendían cruzar a su país.

Bajo este marco asimetría/unilateral, en 1954, inició la Operation Wetback, encabezada por el general Joseph Swing, compañero del presidente Dwight D. Eisenhower en la segunda guerra mundial. Ambos tuvieron experiencias con la zona fronteriza desde 1916-1917, cuando formaron parte de la expedición punitiva que persiguió a Francisco Villa por el estado de Chihuahua. La Operation Wetback, encabezada por Joseph Swing, secreta en su origen, fue una respuesta a los temores de que por la frontera sur de Estados Unidos se introdujeran agentes del comunismo internacional, por lo que el papel de la patrulla fronteriza y de las agencias gubernamentales en su conjunto era detener este proceso. Con dicha operación, cientos de mexicanos, legales o no, fueron investigados y en su caso deportados a México. Muchos de ellos eran nacidos en Estados Unidos, pero desde la construcción en base a la apariencia y el color de piel fueron expulsados del país que nacieron. Las cifras al respecto son variables; lo evidente fue la práctica de la persecución y en su caso la extradición. Cabe señalar que, en ese periodo, el miedo marcó la pauta para que el senador Joseph McCarthy realizara la denuncia de una enorme conspiración comunista. El éxito de su afirmación lo llevó a presidir un comité que se dedicó a acusar de comunistas a periodistas, guionistas de cine, actores y en general todo aquel de quien se sospechara alguna relación con el comunismo. Cabe señalar que Richard Nixon había sido el impulsor de estas persecuciones en años precedentes $y$, como presidente, tomó acciones en ese sentido. A la par, se formó en 1951 la Federal Civil Defense Administration, con la intención de organizar actividades y acciones pertinentes en caso de un ataque soviético. ${ }^{11}$ Paradójicamente, el impulso a las carreteras tuvo un alto impacto en las ciudades de la frontera, ya que facilitó el sistema maquilador ya mencionado, al generarse una infraestructura que permitió la rápida movilidad de insumos y productos manufacturados.

${ }^{11}$ Annual statistic report, Federal civil administration report, 1955. Recuperado de https://www.hsdl.org/?view\&did=34705 [consulta: 20 de diciembre de 2020]. 
De esta forma, la realidad de la zona fronteriza y la mano de obra migrante es bidireccional, es decir, mexicanos que cruzaban formalmente a Estados Unidos bajo la sanción de ambas naciones, y mexicanos que al no cumplir con los requisitos eran trasladados por capataces y empresarios que veían su situación como un punto de vulnerabilidad que les permitía aprovechar el precio bajo de la mano de obra. En sentido contrario, lo que denominamos eje articulador, el tratado de 1944 tenía como una de sus principales acciones vinculantes la construcción de la presa Falcon, es decir, lo que hemos denominado codependencia/negociación. Desde 1946 se redactó la minuta 182 para iniciar las investigaciones para su erección. En 1950 se inició la construcción y en 1953, con la presencia de los presidentes de ambos países, se inauguró una presa que, hasta el día de hoy, es parte de la coordinación cotidiana en la ciLA. Adolfo Ruiz Cortines (1953) enfatizó la amistad entre los dos países. Indicó:

Debemos contribuir a que la atmósfera de crisis que predomina en los asuntos mundiales no divida a los países de este continente. Deseamos que, fieles al pensamiento de nuestros héroes y patricios, resueltos todos a engrandecer nuestras democracias en el ejercicio efectivo de la democracia, permanezcamos unidos en el culto de la soberanía de los pueblos y del derecho inviolable que le asiste al pleno goce de sus libertades civiles y políticas.

Así, Ruiz Cortines remarcaba el tema de la soberanía, en un acto que por la condición del río obligaba a compartir el recurso y los beneficios en la producción de energía. Eisenhower, por su parte, hizo énfasis en la armonía entre las dos naciones. Sin entrar en detalles, indicamos que la presa fue un detonante para la apertura de tierras en los dos países. Desde su planeación, se contempló por ambos gobiernos que era necesario remover pobladores de ambos lados: en el caso de México desapareció Ciudad Guerrero y se fundó Nuevo Guerrero; en Estados Unidos, el poblado de Zapata mantuvo el mismo nombre, pero también fue removido. ${ }^{12}$ Ligado a la presa se construyó, sólo para la parte mexicana, la presa y el canal Anzaldúa, obras que permitieron la apertura de alrededor de 200000 hectáreas al cultivo. Dicho de manera sencilla, la codependencia/negociación redituaba claros beneficios.

${ }^{12}$ El nombre de Zapata se debe a José Antonio de Zapata, quien luchó por la formación de la republica de Río Grande en 1839-1840. 
Eisenhower, en el marco de los temores por el posible ataque soviético y como parte de la preparación para ello -aunque no únicamente-, inició uno de los programas de construcción de carreteras más importantes de Estados Unidos, con The Federal-Aid Highway Act de 1954 (Lewis, 2013; Seely, 1987). ¿Porque es importante para México la reconfiguración de la frontera entre ambos países? Las carreteras, entre sus varios objetivos, permitirían desalojos masivos de las ciudades que eran consideradas objetivos soviéticos. ${ }^{13}$

Por otra parte, durante la segunda guerra mundial, por la necesidad de mano de obra, se promovió el trabajo de las mujeres en la industria. Al finalizar esta, un amplio sector femenino deseaba permanecer en sus puestos de trabajo, aunado al hecho de que los movimientos sindicales se fortalecieron. Con ello, varios sectores empresariales, aprovechando avances en los sistemas productivos y en los medios de transporte, decidieron aprovechar la circunstancia de la excepción fiscal que existía previamente en México e iniciaron el traslado de parte de sus procesos industriales hacia las poblaciones fronterizas. ${ }^{14} \mathrm{Si}$ bien el tema tiene varios antecedentes, destacamos que la excepción fiscal se convirtió en un factor de crecimiento de la industria maquiladora, que tiene como fundamento el ingreso de insumos sin pago de impuestos. El producto elaborado se regresaba a Estados Unidos, donde se comercializaba, incluso, con la posibilidad de retornar a México a través de los mecanismos formales. El esquema estaba relacionado con el trabajo femenino, bajo la premisa de que en nuestro país la mano de obra sería de menor costo (Carrillo y Gomis, 2005). Puerto Rico, como estado libre y asociado, había sido en la década de 1950 el escenario donde esta forma productiva había mostrado los beneficios para el sector empresarial. Para 1971, había alrededor 200 plantas maquiladoras y alrededor de 30000 trabajadoras en las poblaciones de Tijuana, Ciudad Juárez, Matamoros, Nogales y otras.

En 1964 terminó el Programa Bracero, pero no la migración de mexicanos hacia Estados Unidos, ni las operaciones para detener a los ilegales en dicho país. Es frecuente que se relacione el llamado Programa de Industrialización Fronteriza (1965) con el fin del Programa Bracero; sin embargo, sólo en

${ }^{13}$ Annual report, Federal civil administration, 1955. Recuperado de https://training. fema.gov/hiedu/docs/historicalinterest/fcda\%20-\%201955\%20-\%20annual\%20report\%20for\%20 1955.pdf [consulta: 20 de diciembre de 2020].

${ }^{14}$ De manera formal se trataba de la zona libre, implantada desde 1937, pero con muchos antecedentes. Por otra parte, de manera informal, el cruce de mercancías es un tema de vida cotidiana para los habitantes de la frontera mexicana. 
términos discursivos oficiales se puede establecer esa relación, dado que los braceros no fueron quienes mayoritariamente se emplearon en las maquiladoras y, como apuntamos, la mano de obra siguió fluyendo a Estados Unidos. En realidad, fue un nuevo espacio laboral para las mujeres que poco a poco se volvió normal en las ciudades fronterizas.

Los asuntos aquí abordados ilustran lo que consideramos explica y muestra la simultaneidad de la codependencia/negociación, asimetría/unilateral, propuesta que hemos utilizado desde el inicio del presente escrito. Sin embargo, a esta ecuación es necesario agregar otros temas en los que pretendemos utilizar esta paradoja que consideramos ilustra la relación en la zona donde inicia, desde la perspectiva Norte-Sur, América Latina. En este escenario no hemos abordado las aguas subterráneas, la diversidad medioambiental, el turismo, el gas natural o la relación de la frontera con el Golfo de México y el de California. Tampoco el intenso comercio formal e informal que se genera de manera cotidiana o la construcción, cerca al límite fronterizo, de instalaciones militares de gran importancia. Sólo tocamos parte del proceso para demostrar la relación y la necesidad de entender los vínculos, paradójicos y complementarios, en un límite internacional que obliga a plantear esquemas diferentes para su comprensión.

\section{NEGOCIAR LA FRONTERA, UNA CARA AL MUNDO (PERO SÓLO UNA)}

La guerra fría, como concepto construido para diferenciar dos posturas contrapuestas sobre la organización de la producción, tuvo muchos escenarios de manera simultánea. La Alianza para el Progreso, como política específica, llevaba la pretensión de confrontar la presencia de grupos y organizaciones que simpatizaban con la URSS y el comunismo. Por otra parte, las necesidades específicas de la extensa frontera y, para este caso, un tema de orden geológico planteaba necesidades de cooperación dentro de la codependencia/ negociación.

La Alianza para el Progreso lanzada por John F. Kennedy y continuada por Lyndon B. Johnson tuvo como eje discursivo indicar que se pretendía mejorar las condiciones de vida en los países de América Latina, con la inversión de 20000 millones de dólares. Sin duda, era una respuesta a la enorme simpatía generada por la revolución cubana y el fracaso en bahía de Cochinos 
(Jones, 2008). Dicho operativo, preparado desde la administración de Eisenhower, fue un intento fallido de recuperar el control de la isla. En ese contexto, Kennedy reconoció una decisión que databa de 1911, mismo que había quedado pendiente por medio siglo. Nos referimos al tema de El Chamizal, mismo que tomó un valor simbólico para configurar la buena relación entre ambos países. De manera muy sintética, planteamos que por condiciones naturales del río Bravo, desde 1857, este se movió paulatinamente hacia el sur, en beneficio de la ampliación de territorio estadunidense y en detrimento de la actual Ciudad Juárez, Chihuahua. ${ }^{15}$ A principios del siglo xx, el caso se sometió a la decisión de Eugene Lafleur, miembro de la International Joint Commission, integrada por Estados Unidos y Canadá. Dicha comisión era semejante a la Comisión Internacional de Límites entre México y Estados Unidos. En 1911, Lafleur dio un fallo en el sentido de dividir la propiedad de El Chamizal. Con ello, ninguno ganó el caso, pero en la división, México obtuvo mayor cantidad de hectáreas. Estados Unidos estuvo en desacuerdo con la decisión y argumentó que se recurrió al arbitraje para que decidiera sobre todo El Chamizal, no para dividirlo, por lo que no aceptaron el fallo. El caso se mantuvo como parte de los conflictos y negociaciones entre los dos países durante medio siglo (Bustamante, 1999; Cámara de Diputados, 2014; United States Senate, 1964). Entre 1961 y 1964 se realizaron negociaciones de las que, finalmente, se acordó regresar a México la mayor parte de este. La resistencia a la medida fue $-\mathrm{y}$ sigue siendo- expresada por los paceños de diversas formas, dado que para ellos la zona era parte de la ciudad y se habían construido escuelas, hospitales, hoteles y zonas habitacionales.

El tema es parte de los que hemos denominado codependencia/negociación. Hasta la fecha, el asunto es citado por la diplomacia mexicana como uno de los triunfos más destacables, basados en principios de derecho internacional (Cámara de Diputados, 2014). Sin embargo, más allá de los argumentos, debe entenderse como la prueba de buena voluntad por parte de Kennedy para mostrar sus intenciones en un caso donde salía directamente afectado un sector de la población de El Paso, Texas. Era una forma de negociar con todos los países del continente americano y poner en evidencia su intención de una buena relación hemisférica. ¿Qué perdían los estadunidenses con ceder un

15 El nombre en ese momento era Paso del Norte. 
espacio de 177 hectáreas $?^{16}$ En cambio, demostraban que Estados Unidos era un aliado y, por tanto, buscaba generar antagonismos con la URSS. En los años siguientes, con Lyndon B. Johnson y Gustavo Díaz Ordaz como presidentes, se realizó la ceremonia como la muestra de amistad entre ambas naciones y la modificación del espacio limítrofe. En estas negociaciones, ¿hubo algún reclamo de Adolfo López Mateos a Kennedy por la ambivalencia de la Operation Wetback y el Programa Bracero? En los eventos realizados entre Johnson y Díaz Ordaz para celebrar el acuerdo, ¿se analizaron las condiciones de los trabajadores?, ¿se trató el tema de la revolución cubana y la postura de México, comentada anteriormente? La respuesta es negativa. En cambio, entre 1966 y 1969 se realizó una inversión de casi 90000000 de dólares para transformar la zona de El Chamizal, así como los cruces fronterizos. Se construyeron dos puentes y obras para recanalizar el agua del río Bravo. Johnson, por su parte, en 1968, el día de la inauguración destacó que la relación entre los dos países era tan buena que no era necesario tener guardias para cuidar el límite. En ese contexto, se realizaron pláticas para solucionar otro conflicto por límites en la zona de Ojinaga, Chihuahua y Presidio, Texas. El tema llevó a la firma del tratado para resolver las diferencias fronterizas pendientes y mantener a los ríos Bravo y Colorado como frontera internacional entre México y Estados Unidos. ${ }^{17}$ Esta firma la realizaron Richard Nixon y Gustavo Díaz Ordaz, en vísperas de la toma de la presidencia de Luis Echeverría.

Además, simultáneamente, la cILA se coordinaba para construir, en concordancia con el tratado de 1944, la presa de La Amistad, ubicada en el límite fronterizo entre Coahuila y Texas. En 1958 -en la minuta 207- se iniciaron las recomendaciones para su localización. En 1963 se empezó con la cimentación y, en 1969, se inauguró, de nuevo, con la presencia de los presidentes de cada nación.

A ello nos referimos cuando, desde el principio de este escrito, señalamos que la lógica de la construcción del límite no corresponde a las mismas líneas que las publicitadas por los gobiernos que para esas fechas aún se

${ }^{16}$ La cantidad de hectáreas no corresponde al laudo de 1911 dado que se involucró el Corte de Córdoba, aledaño a la zona, para reconstituir toda el área, con la intención de hacerla funcional a los cruces fronterizos. Al respecto, se puede consultar la minuta 214, firmada el 28 de agosto de 1963. http://www.cila.gob.mx/actas/214.pdf [consulta: 3 de enero de 2021].

${ }^{17}$ El tratado es de suma importancia, no sólo para la zona limítrofe terrestre, sino que fue parte importante para el establecimiento de la frontera marítima. En ese momento, 1970, bajo la idea de la Convención de Ginebra sobre el Mar Territorial y la Zona Contigua. 
autolegitimaban revolucionarios, para el caso mexicano. En este caso, con el ingreso de Nixon a la presidencia, la idea que hemos planteado, codependencia/negociación, simultánea a asimetría/unilateral se hace evidente en corto plazo. Así, en 1969, Richard Nixon, como promesa de campaña iniciaba el largo proceso de retirar las tropas de Vietnam y planteaba que México no realizaba el esfuerzo suficiente para controlar el contrabando de drogas, en particular la marihuana. La Operación Intercepción, organizada de manera secreta, fue una medida que se implementó para hacer revisiones minuciosas entre el 21 de septiembre y el 11 de octubre de 1969. Si bien las reclamaciones mexicanas -y de estadunidenses afectados también- fueron escuchadas, el tema permite ilustrar la complejidad de la relación (Brecher, 1972; Gooberman, 1974; Ojeda, 1978, p. 304). Al parecer, Díaz Ordaz fue advertido en la inauguración de la presa La Amistad de que se realizaría el operativo. Sin embargo, no hubo reclamos ni políticas específicas desde el poder presidencial.

De manera paralela a la construcción de la presa La Amistad y lo referente a El Chamizal, en el río Colorado se inició, desde 1961, el denominado convencionalmente problema de la salinidad del río Colorado. ${ }^{18}$ Sin entrar en los detalles técnicos, sintetizamos que durante poco más de una década, los agricultores del Valle de Mexicali vieron afectadas sus tierras debido a un incremento sustancial en la salinidad de las aguas que se recibían. Si bien el tema era de importancia desde años atrás, el incremento se debió a que en el valle de Wellton-Mohawk en Arizona, el Bureau of Reclamation bombeaba agua de drenaje hacia el río, sumando dicha agua como parte de la cuota que debía entregar a México (1 850.234 millones de m3). Así, mientras en el Bravo se presentaban las condiciones de cooperación ya comentadas, en el Colorado el tema se tornaba un conflicto internacional (Ward, 2003, pp. 55-70). Además, actores políticos como Lázaro Cárdenas intervinieron con el fin de sumarse al sector campesino del Valle de Mexicali y presionar para una solución al conflicto. McGeorge Bundy, asistente especial para asuntos de seguridad nacional de Estados Unidos, al explicarle el tema a Lyndon B. Johnson, señaló que solucionar el problema era relevante, dado que para Estados Unidos la relación con México era un ejemplo de su postura ante el mundo. ${ }^{19}$

${ }^{18}$ Señalamos convencionalmente porque la denominación salinidad del río Colorado deja de lado problemas más complejos que requieren ser observados en otra temporalidad que no corresponde a la propia de este escrito.

${ }^{19}$ Foreign Relations of the United States Johnson Library, National Security File, Country File, Mexico, vol. I, Cables, 12/63-12/65. Confidential. 
Es decir, negociar el asunto con nuestro país -semejante a El Chamizal- era también una carta de presentación para otros asuntos de orden internacional. Dicha afirmación es parte de lo que hemos apuntado con respecto al tratado de 1944, incomprensible para algunos sectores -sobre todo de Estados Unidos- dado que México recibe cuatro veces más agua de la que entrega. Es decir, negociar la reconstitución del límite fronterizo era parte de la imagen que Estados Unidos requería para demostrar la buena voluntad de sus acciones, lo que le permitía proponer -y de acuerdo con el caso imponer-sus políticas en otras partes del mundo. Así, las necesidades de la extensa región fronteriza responden a demandas en el orden local, que generalmente es binacional, y, por otro, dada la misma colindancia, se creó un discurso nacionalista que remarcó el principio de autodeterminación de los pueblos, pero se respondía a las necesidades fronterizas y se mantenía fuera del discurso oficial el tema de la mano de obra y las violaciones a los derechos humanos de los trabajadores migrantes.

Cabe señalar que las negociaciones sobre el tema de las aguas de drenaje de Wellton-Mohawk conllevó a llamados para la solidaridad hemisférica. Sintetizamos el tema al indicar que la firma de las minutas 218 en 1965 y la 242 en 1972 plantearon una solución que, como es frecuente, generó nuevas tensiones en años posteriores. Como parte de los acuerdos, se construyó un dren para que dichas aguas fueran conducidas al golfo de California (Bustamante, 1999, pp. 339-363). Paradójicamente, con ello se dio vida a uno de los humedales de suma importancia en el bajo delta del río Colorado, como es la ciénega de Santa Clara, misma que hasta la fecha depende, en territorio mexicano, de las mencionadas aguas salitrosas (Glenn, Felger, Burquez y Turner, 1992; Glenn, García y Tanner, 1999).

Así, la minuta 242 se convirtió, luego de una década, en un referente de las buenas relaciones entre los dos países. Para sumar a ello, indicamos que el crecimiento paulatino de las maquiladoras a inicios de la década de 1970 y la necesidad de trasladar agua hacia la ciudad de Tijuana, llevó a otro de los acuerdos que hasta la fecha se mantiene. La colaboración estadunidense consiste en permitir que a través del acueducto que transporta el agua del río Colorado hacia Los Ángeles y San Diego, California, ciudad inmediata en la frontera, sea el mismo por el cual se traslade agua a Tijuana y Tecate. Así, la minuta 240, firmada en 1972, inició una condición que permanece hasta nuestros días. El gobierno del estado de Baja California, encargado del abasto urbano -no los municipios- solicitan a la CILA, sección mexicana, que se 
recurra al concepto de entregas de emergencia. Se firma un acta y se aprovechan las instalaciones de Estados Unidos, mismas que mediante el cobro respectivo, realizan el traslado. ${ }^{20}$

Otro ejemplo de la propuesta de codependencia/negociación es el referente a las instituciones académicas estadunidenses que apoyaron el surgimiento de centros de investigación en territorio de México, particularmente en la península de Baja California y por extensión el golfo de California. En el siglo $\mathrm{xx}$ se han elaborado numerosos estudios acerca de una condición natural que tiene profunda relación con la vertiente del Pacífico y que vincula a California, Estados Unidos, con la península. Nos referimos a la denominada falla de San Andrés, con toda una serie de fallas de menores proporciones (Cruz, 2002). Una de las referencias es que cada año la península de Baja California se desprende seis centímetros, debido a que se localiza sobre la denominada placa del Pacífico. Por su parte, el golfo de California es reconocido como el acuario del mundo por su biodiversidad. ${ }^{21}$ Organismos como la Scripps Institution of Oceanography, ubicado en San Diego, California, o la Universidad de Arizona, fueron clave para la apertura de centros de investigación en la entidad. Por su parte, México tenía especial interés en el tema del golfo, dado que desde el tratado de Guadalupe-Hidalgo había quedado establecido que las embarcaciones de Estados Unidos podían utilizarlo para la navegación. Con las discusiones sobre el mar patrimonial, generadas sobre todo por países africanos y sudamericanos, el asunto cobró particular relevancia tanto para la seguridad nacional estadunidense, por el temor a los submarinos soviéticos, como por la investigación científica tanto del golfo como por la falla geológica mencionada (Samaniego, 2015). De esta forma, la Universidad Autónoma de Baja California -fundada oficialmente en 1957tuvo su primer programa de licenciatura ligado a las ciencias marinas con el apoyo de instituciones de California. De igual forma, el Consejo Nacional de Ciencia y Tecnología, creado en 1970, fundó el Centro de Investigación

${ }^{20}$ Cabe señalar que, a pesar de las manifestaciones de Donald J. Trump, como candidato y presidente de Estados Unidos, esta condición se mantiene a través de la minuta 322. Cabe apuntar que también en temas de saneamiento entre las ciudades existen planes de orden binacional, generalmente bajo la supervisión de la cilA, que responden a las necesidades regionales. Así, por ejemplo, el United States-Mexican-Canada Agreement (USMCA) que sustituye al Tratado de Libre Comercio de 1994, se contempla la inversión de 300000000 de dólares para el área del río Tijuana, que desemboca en Imperial Beach, al sur de San Diego, California.

${ }^{21}$ El golfo de California o Mar de Cortés involucra a los estados mexicanos de Sonora, Sinaloa, Nayarit, Baja California Sur y Baja California. 
Científica y de Educación Superior de Ensenada (CICESE). Ambas instituciones en estrecha relación con la Scripps Institution of Oceanography. De igual forma, se fundó el Observatorio Astronómico Nacional en la sierra de San Pedro Mártir, sitio elegido tanto por investigadores de la Universidad Nacional Autónoma de México como de la Universidad de Arizona (Álvarez y López, 1986). La relación con dicha universidad ha sido fundamental para el desarrollo de la investigación. Este ejemplo permite contextualizar la relación a la que aludimos como paradójica, pero que es necesaria para ubicar, parcialmente dados los otros temas que no abordamos en este escrito, el significado de una relación durante el proceso denominado como guerra fría.

\section{CONCLUSIONES}

Las tensiones internacionales son parte de la historia de la construcción de la frontera entre México y Estados Unidos. Es un límite que, desde la concepción estadunidense, no se circunscribe a la relación con su vecino del sur. La relación de poder se manifiesta de diversas maneras, pero no es omnipotente ni omnipresente. Existen necesidades y por tanto debilidades que establecen una relación que hemos ubicado en dos sentidos a lo largo del presente escrito, codependencia/negociación-asimetría/unilateral. Bajo este marco es posible entender temas centrales en una relación que dista mucho de ser entre iguales, pero que se torna necesaria para ambas partes. El tema de las cuencas internacionales es probablemente el más evidente, dado que tiene significado en todos los ámbitos.

Si América Latina inicia en la frontera México-Estados Unidos, consideramos que la observación aquí planteada lleva a reflexionar sobre la necesidad de ubicar la relación en función de las necesidades de construcción de un espacio que no se apega a lo indicado por el discurso que destaca una separación de la nación que, sin duda, desempeña un papel hegemónico en el continente. Como apuntamos al inicio del escrito, una de las tesis predominantes en el análisis como la de Mario Ojeda, si bien permite entender parte de la explicación, no profundiza en la codependencia/negociación que implica en la vida cotidiana de varios millones de personas en ambos lados de la frontera. Pero, además, esta relación no termina en el límite, sino que está condicionada, para Estados Unidos, a poco más de 2000 kilómetros en su interior, dado que, desde Wyoming, Colorado y Utah, surge la fuente de agua 
que es utilizada por más de 2000000 de personas en Baja California/San Luis Río Colorado, Sonora, y para el caso mexicano desde 900 kilómetros, longitud del río Conchos.

Para Estados Unidos, demostrar una política rígida con respecto a la migración es satisfactoria para los temores generados ante posibles ataques de sus enemigos a través de la frontera con México. Esta tensión se puede observar desde finales del siglo xIx, pero en el xx cobró particular importancia en contra de los japoneses, italianos, alemanes y, posteriormente, el comunismo soviético. Por ello, la construcción de barreras es una propuesta que se repite desde las primeras décadas del siglo xx. Sin embargo, la migración es conveniente para empresarios y agricultores que dependen de esa mano de obra desde hace más de un siglo. Por ello, la tensión lleva a medidas unilaterales que son ubicados con facilidad, debido a la vulnerabilidad tanto del migrante como al hecho de que México en el largo plazo se ha caracterizado por ser un país expulsor.

Así empieza el territorio que convencionalmente se conoce como América Latina. ¿Separados?, ¿en colaboración?, ¿dos culturas distintas? La referencia a la frontera México-Estados Unidos, por algunos, destaca una separación, mientras que otros hablan de una integración a partir del acuerdo comercial de 1994, mismo que en México es ubicado como Tratado y en Estados Unidos y Canadá como Acuerdo. Sin embargo, como aquí consideramos haber demostrado, la relación es más compleja, con interdependencia y conveniencia para ambos, con asimetrías notables y con un espacio limítrofe que, a su vez, presenta abordajes distintos de acuerdo con necesidades de una zona en la que el termino seguridad siempre aparece, como un asunto vinculado al contexto internacional.

A lo largo de este escrito se han mencionado términos como espacio fronterizo, límite fronterizo y frontera México-Estados Unidos. Su uso parte de un problema que consideramos no se ha abordado a pesar de que se citan en numerosos trabajos y conceptos sobre frontera. Es decir, a pesar de las numerosas propuestas, el tema del espacio de influencia queda poco explicado. Por ello, planteamos como pregunta: ¿qué es el espacio fronterizo?, ¿es lo mismo que el límite? La respuesta apunta a que no es así. Por ello la referencia a las "varias fronteras" que hemos mencionado. En ese sentido, quedan muchos aspectos por abordarse en un periodo de la historia del mundo en el que, el espacio fronterizo, fue central para la concepción de políticas de orden internacional. 


\section{LISTA DE REFERENCIAS}

Agnew, J. (2005). Hegemony: the new shape of global power. Philadelphia: Temple University Press.

Álvarez, C. J. (2019). Border land, border water: A history of construction on the U.S.Mexico divide. Austin: University of Texas Press.

Álvarez, M. y López, E. (1986). Los últimos diez años del Observatorio Astronómico Nacional. En M. A. Moreno Corral (coord.), Historia de la astronomía en México. México: Fondo de Cultura Económica.

Anderson, M. (1996). Frontiers: territory and state formation in the modern world. Oxford: Polity.

Avella, I. (2016). El impacto del tratado comercial de 1942 entre México y Estados Unidos en el flujo comercial bilateral (1943-1950). En M. Lopes y C. Zuleta (coords.), Mercados en común. Estudios sobre conexiones, negocios y diplomacia en las Américas (siglos XIX y Xx) (pp. 193-226). México: El Colegio de México.

Avella, I. (2018). La comercialización del petróleo entre México y Estados Unidos en el marco del tratado comercial de 1942. Historia Mexicana, 67(4), 1725-1778. DoI: https://doi.org/10.24201/hm.v67i4.3568

Bethell, L. (2016). Brasil y América Latina. Istor, 67, 109-145.

Brands, H. (2010). Latin America's cold war. Cambridge: Harvard University Press.

Brecher, E. (1972). The 1969 marijuana shortage and Operation Intercept. The Consumers Union Report on Licit and Illicit Drugs. Reports Magazine. Recuperado de https://www.druglibrary.org/schaffer/library/studies/cu/CU59.html

Bustamante, J. (1989). Frontera México-Estados Unidos: reflexiones para un marco teórico. Frontera Norte, 1, 17-24.

Bustamante, J. (1999). La Comisión Internacional de Límites y Aguas entre México y Estados Unidos, sus orígenes y su actuación hasta 1996. Ciudad Juárez, Chihuahua: Universidad Autónoma de Ciudad Juárez/San Diego State University/New Mexico State University.

Cámara de Diputados (2014). El Chamizal. A 50 años de su devolución. México: LXII Legislatura.

Cárdenas, E. (1996). La política económica en México, 1950-1990. México: El Colegio de México/Fondo de Cultura Económica.

Carrillo, J. (1985). Conflictos laborales en la industria maquiladora. Tijuana, Baja California: Centro de Estudios Fronterizos del Norte de México.

Carrillo, J. y Redi Gomis (2005). Generaciones de maquiladoras: un primer acercamiento a su medición. Frontera Norte, 17(33), 25-51. 
Chacón, S. (2011). La relación entre México y Estados Unidos (1940-1955): entre el conflicto y la cooperación. España: Fondo de Cultura Económica.

Cruz, M. (2002). Catálogo de las fallas regionales activas en el norte de la Baja California, México. GEOS, 22, 37-42.

Enríquez, E. (1976). El tratado entre México y los Estados Unidos de América sobre ríos internacionales. Una lucha nacional de noventa años. México: Facultad de Ciencias Políticas y Sociales-Universidad Nacional Autónoma de México.

Gaddis, J. (1972). The United States and the origins of the cold war, 1941-1947. Nueva York: Columbia University Press.

Glenn, E., Felger, R., Burquez, A. y Turner, D. (1992). Cienega de Santa Clara: endangered wetland in the Colorado River delta, Sonora, México. Natural Resources Journal, 32, 817-824.

Glenn, E., García, J. y Tanner, R. (1999). Status of wetlands supported by agricultural drainage water in the Colorado River delta, Mexico. Horticultural Science, 34(1), $39-45$.

Gooberman, L. (1974). Operation Intercept: The multiple consequences of public policy. Nueva York: Pergamon Press.

Hatch, G. (2017). Paso del Norte. La competencia por las aguas subterráneas transfronterizas. México: El Colegio de Chihuahua/Universidad Autónoma de Ciudad Juárez.

Hoddeson, L., Henriksen, P., Meade, R. y Westfall, L. (1993). Critical assembly: A technical history of Los Alamos during the Oppenheimer years, 1943-1945. Nueva York: Cambridge University Press.

Hundley, C. (1963). The dividing waters: Mexican American controversies over the waters of the Colorado River and The Rio Grande, 1880-1960. Los Ángeles, California: University of California.

Jones, H. (2008). The Bay of Pigs. Nueva York: Oxford University Press.

Kissinger, H. (2003). Ending the Vietnam war: A history of America's involvement in and extrication from the Vietnam war. Nueva York: Simon \& Schuster.

LaFeber, W. (1972). America, Russia, and the cold war, 1945-1971. Nueva York: Wiley.

Leffler, M. (1992). A preponderance of power: National security, the Truman administration, and the cold war. Stanford: Stanford University Press.

Lewis, T. (2013). Divided highways. Building the interstate highways. Transforming American life. Cornell University Press.

Loaeza, S. (2016). La fractura mexicana y el golpe de 1954 en Guatemala. Historia Mexicana, 66(2), 725-791. DoI: https://doi.org/10.24201/hm.v66i2.3346

Lytle, K. (2010). Migra! A history of the U.S. Border Patrol. Berkeley: University of California Press. 
Menchaca, M. (2011). Naturalizing Mexican immigrants: A Texas history. Austin: University of Texas Press.

Meyer, L. (2010). Relaciones México-Estados Unidos. Arquitectura y montajes de las pautas de la guerra fría, 1945-1964. Foro Internacional, 50(2), 202-242. Recuperado de http://forointernacional.colmex.mx/index.php/fi/article/view/1991

Mignolo, W. D., (2005). The idea of Latin America. Oxford: Blackwell.

Mikoyan, S. (2012). The Soviet Cuban missile crisis: Castro, Mikoyan, Kennedy, Khrushchev, and the missiles of november. Washington, D. C.: Woodrow Wilson Center Press/California: Stanford University Press.

Moya, J. (2016). América Latina como categoría histórica en una perspectiva global. Istor, 67, 16-59.

Munguía, A. (2015). La política migratoria en los Estados Unidos. México: Benemérita Universidad Autónoma de Puebla.

Ojeda, M. (1976). Alcances y límites de la política exterior de México. México: El Colegio de México.

Ojeda, M. (1978). Cuestiones clave en las relaciones México-Estados Unidos. Foro Internacional, 19(2), 303-325. Recuperado de https://forointernacional.colmex. $\mathrm{mx} /$ index.php/fi/article/view/2348

Orozco, C. (2009). No Mexicans, women, or sdogs allowed: The rise of the Mexican American Civil Rights Movement. Austin: University of Texas Press.

Paredes, A. (2004). La Operación Cóndor y la guerra fría. Universum, 1(19), 122-137. Dor: http://dx.doi.org/10.4067/S0718-23762004000100007

Pettinà, V. (2016). ¡Bienvenido Mr. Mikoyanj: tacos y tractores a la sombra del acercamiento soviético-mexicano, 1958-1964. Historia Mexicana, 66(2), 793-852. DoI: https://doi.org/10.24201/hm.v66i2.3347

Pettinà, V. (2018). Historia mínima de la guerra fría en América Latina. México: El Colegio de México.

Pisani D. (1992). To reclaim a divided west. Water, law, and public policy, 1848-1902. Albuquerque: University of New Mexico Press.

Pisani, D. (1996). Water, land, and law in the west. The limits of public policy, 1850-1920. Lawrence, KS: University Press of Kansas.

Powaski, R. (1998). La guerra fría. Estados Unidos y la Unión Soviética, 1917-1991. Barcelona: Crítica.

Prados, J. (2009). Vietnam: The history of an unwinnable war, 1945-1975. Lawrence, KS: University Press of Kansas.

Ruiz, A. (19 de octubre, 1953). Memoria política de México. Recuperado de https:// www.memoriapoliticademexico.org/Textos/6Revolucion/1953IPF.html 
Saunders, F. (2001). La cia y la guerra fría cultural. Madrid: Editorial Debate.

Samaniego, M. (2006). Ríos internacionales entre México y Estados Unidos. Los tratados de 1906 y 1944. México: El Colegio de México/Universidad Autónoma de Baja California.

Samaniego, M. (2012). Cuencas internacionales y usos sociales del agua. Formación de espacio de cooperación y conflicto: el norte de México y oeste de Estados Unidos. Secuencia, 83, 147-177. DoI: https://doi.org/10.18234/secuencia.v0i83.1158

Samaniego, M. (2015). Instituciones, ciencia y poder en Baja California. Relaciones sociales para la construcción del conocimiento. En E. González y C. Castro (coords.), Instituciones, ciencia, tecnología y vinculación en el noroeste de México. México: Centro de Investigaciones Biológicas del Noroeste.

Samaniego, M. (2018). Ríos internacionales, historiografía y presentismo. En R. Ríos (coord.), Atisbos de nación y memoria desde la historia y los estudios culturales. México: Universidad Autónoma de Baja California.

Seely, B. (1987). Building the American highway system, engineers and policy making. Philadelphia: Temple University Press.

Selser, G. (1982). Las bases de EE. UU. en Panamá. El destino del Comando Sur y de la Escuela de las Américas. Nueva Sociedad, 63, 57-74.

Summers, H. (1981). On strategy. The Vietnam war in context. Strategic Studies Institute.

Strange, S. (2002). La retirada del Estado. La difusión del poder en la economía mundial. Barcelona, España: Intermón Oxfam Editorial.

United States Senate (1964). Compliance with convention on The Chamizal. Hearing before the committee on foreign relations, 68th. Congress, $2^{\text {a }}$. Session, on S. 2394. Washington: United States Government Printing Office,

Ward, E. (2003). Border Oasis: Water and the political ecology of the Colorado river delta, 1940-1975 (La frontera: People and their environments in the US-Mexico borderlands). Tucson: University of Arizona Press.

Zamora, E. (2003), The world of the Mexican worker in Texas, Texas A \& M, University Press. 